\title{
Effects of an external magnetic field, and of oblique radio-frequency electric fields on multipactor discharge on a dielectric
}

\author{
A. Valfells, L. K. Ang, Y. Y. Lau, and R. M. Gilgenbach \\ Department of Nuclear Engineering and Radiological Sciences, University of Michigan, Ann Arbor, \\ Michigan 48109-2104
}

(Received 4 June 1999; accepted 27 October 1999)

\begin{abstract}
This paper analyzes, separately, the effects of an external magnetic field, the rf magnetic field, and of an oblique rf electric field, on multipactor discharge on a dielectric. Using Monte Carlo simulation, we obtain the susceptibility diagram in terms of the magnetic field, the rf electric field, and the dc charging field for various dielectric materials. We find that a magnetic field parallel to either the rf electric field or the dc electric field does not qualitatively change the susceptibility diagram. However, an external magnetic field perpendicular to both the rf electric field and the dc electric field can significantly affect the susceptibility diagram. Thus oriented magnetic fields lower the upper susceptibility bound when the magnetic field strength is approximately equal to $B_{\text {res }}[T]$ $=0.036 f(\mathrm{GHz})$, where $f$ is the $\mathrm{rf}$ frequency. Both the lower and upper susceptibility boundary may be raised significantly by a large external magnetic field, $B \gg B_{\text {res }}$. Susceptibility to single surface multipactor is greatest when the rf electric field is nearly parallel to the dielectric, but is dramatically decreased for angles of obliqueness greater than approximately $5^{\circ}-10^{\circ}$. The $\mathrm{rf}$ magnetic field does not affect the lower boundary, but may extend the upper boundary greatly. (C) 2000 American Institute of Physics. [S1070-664X(00)03002-0]
\end{abstract}

\section{INTRODUCTION}

Multipactor discharge is an ubiquitous phenomenon observed in a multitude of devices that employ microwaves. ${ }^{1}$ It may occur when a metallic gap or dielectric surface is exposed to a rf electric field under some favorable conditions. Its avoidance has been a major concern among workers on high power microwave sources, rf accelerators, and spacebased communication systems. ${ }^{1-8}$

For the past few years, multipactor discharge in a metallic gap has been studied with regards to its evolution, ${ }^{7,9,10-12}$ its saturation level, ${ }^{13}$ its dependence on materials, ${ }^{13}$ and its frequency response. ${ }^{14}$ Recently, we extended our studies to multipactor discharge on a dielectric surface, including particle simulations ${ }^{15}$ and a dynamic theory. ${ }^{16}$ In contrast to the two-surface multipactor discharge on metallic gap, multipactor discharge on dielectric is a single-surface phenomenon, and it is more likely to occur. ${ }^{15}$ It was found that the power deposited on a dielectric by multipactor discharge is on the order of $1 \%$, or less, of the rf power over a wide range of operating conditions and materials, and the saturation mechanism is due to space charge effects. ${ }^{16}$ All of these calculations omit the effect of outgassing and subsequent ionization by the multipacting electrons. We shall continue this assumption in this paper.

rf window breakdown (or dielectric failure $)^{2-5,15-17}$ has been a limiting factor in many high power microwave systems. In general, an avalanche of secondary electrons caused by multipactor discharge occurs in the prebreakdown phase. When this avalanche of electrons reaches a sufficiently high saturation level, it induces gas outgassing from the dielectric surface. Further ionization caused by these electrons provides a gaseous-like discharge (sometimes called flashover) within the desorbed gas layer, and it eventually turns into the breakdown phase of the dielectric. Due to the high susceptibility of multipactor discharge on dielectric, ${ }^{8,15,16}$ a small amount of seed electrons may grow to a high level that will initiate the breakdown of dielectric. To prevent the breakdown, it is necessary to suppress or eliminate the initial multipactor discharge, before catastrophic failure.

In this paper, we set our sights on susceptibility to multipactor discharge on a dielectric. ${ }^{15}$ Our main interest is, thus, initiation of the discharge, and not subsequent development of it, although we have previously shown that the susceptibility diagram may be used to estimate the saturation level of the discharge. ${ }^{16}$ Therefore instead of a dynamic theory, where the restoring electric field changes via secondary emission, we use particle simulations with a fixed restoring field. In this paper we extend our simulations to obtain more general susceptibility diagrams. First, we include the effects of a constant external magnetic field, varying the field strength over a wide range and for different directions. We also briefly examine the effects of the rf magnetic field. ${ }^{18}$ Second, we will remove the external magnetic field but allow the rf electric field to have an arbitrary angle with the surface of the dielectric. This extension is necessary since the rf electric field on a rf window is not necessarily parallel to the window surface. These variations alter the trajectory of the secondary electrons emitted from the surface, changing the characteristics of the secondary electron avalanche along the surface. Our motivation for this study is threefold. Since multipactor discharge on dielectric is more susceptible than that on metal, we are particularly interested in examining the possibility of inhibiting multipactor discharge by an external magnetic field. Previously we considered only the case 
where the rf electric field is parallel to the dielectric. On a rf window, this idealization need not be true. We also use the results for the external magnetic field to infer the importance of the rf magnetic field upon the multipactor discharge. By allowing an arbitrary angle, $\psi$, between the rf electric field and the dielectric we would like to gain an understanding of the effects of different EM wave modes upon susceptibility to multipactor discharge. To isolate the effects of external magnetic field from the oblique electric field, we treat these effects separately. Three different magnetic field configurations are considered: (a) parallel to rf field, (b) parallel to dc charging field, and (c) perpendicular to both rf and dc fields. The angle of obliqueness, $\psi$, for the rf field is varied from 0 (parallel to dielectric) to $90^{\circ}$ (normal to surface). It should be noted that when one determines the angle of obliqueness, $\psi$, one must consider reflection from the dielectric.

In Sec. II, we describe the model used for multipactor discharge. In Sec. III we review prior results and comment on the structure of the susceptibility diagram. In Sec. IV, we examine magnetic field effects, present the susceptibility diagram, and discuss the underlying physics involved. In Sec. $\mathrm{V}$, we present the results for oblique rf fields, explaining the variations of the susceptibility diagram, and explore possible implications. Finally, some concluding remarks are given in Sec. VI.

\section{MODEL AND ANALYSIS}

We use a transmission line model ${ }^{16}$ to investigate both the effects of external electric field and oblique rf electric field. The geometry for the case of an external magnetic field is shown in Fig. 1. On the dielectric surface there is a dc dielectric charging electric field, $\mathbf{E}_{\mathrm{dc}}$, a rf electric field, $\mathbf{E}_{\mathrm{rf}}$ (with amplitude $E_{\mathrm{rf} 0}$, frequency $\omega$, initial phase $\theta$, and angle of obliqueness $\psi, \psi=0$ in Fig. 1), and an external magnetic field, B (Fig. 1). We always set the normal charging field, $\mathbf{E}_{\mathrm{dc}}$, along the $x$ axis, and the rf electric field in the $x-y$ plane. Note that for the purpose of our analysis we allow the angle, $\psi$, between the rf electric field and the dielectric to be nonzero if and only if $\mathbf{B}$ vanishes. Electrons emitted with a random velocity, $\mathrm{v}_{0}$, and a random angle, $\phi$ with respect to the $y$ axis are subjected to forces imposed by $\mathbf{E}_{\mathrm{dc}}, \mathbf{E}_{\mathrm{rf}}$, and B. Having gained energy from $\mathbf{E}_{\mathrm{rf}}$, the electrons strike the surface with impact energy $E_{i}$, and impact angle, $\xi$. When the impact energy lies between $E_{1}$ and $E_{2}$, the first and second crossover energy, respectively, of the secondary electron yield curve (see Fig. 2), the ratio of secondary electrons emitted from the surface to primary electrons incident upon it, $\delta$, will be greater than unity. In this case we can have a net gain of secondary electrons occur.

The dependence of the secondary electron yield on the impact energy and impact angle is adopted from Vaughan's empirical formula ${ }^{19}$

$$
\delta=\delta\left(E_{i}\right)=\delta_{\max }\left(w e^{1-w}\right)^{k},
$$

where $\delta_{\max }$ is the maximum value of $\delta, w=E_{i} / E_{\max }$, $E_{\max }$ being the impact energy which yields $\delta_{\max }$, and $k$

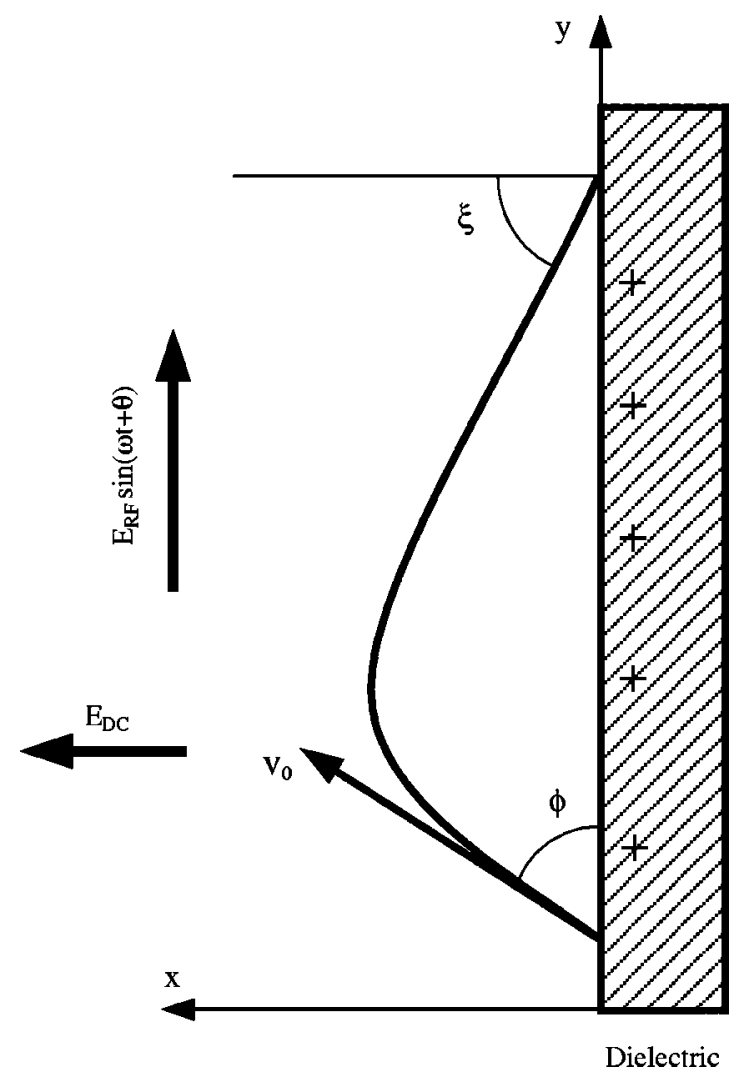

FIG. 1. Schematic of a single-surface multipactor in a parallel rf, dc electric field, and external magnetic field perpendicular to $\mathrm{rf}$ and dc electric field. Angle of obliqueness, $\psi=0$.

$=0.62$ for $w<1$ and $k>0.25$ for $w>1$. For the impact angle, $\xi, E_{\max }$ and $\delta_{\max }$ are adjusted according to the following equations:

$$
\begin{aligned}
& E_{\max }=E_{\max 0}\left(1+\frac{k_{s} \xi^{2}}{\pi}\right), \\
& \delta_{\max }=\delta_{\max 0}\left(1+\frac{k_{s} \xi^{2}}{2 \pi}\right) .
\end{aligned}
$$

Here $E_{\max 0}$ and $\delta_{\max 0}$ are the parameters for an impact angle of zero (i.e., normal to the surface), and $k_{s}$ is a surface smoothness factor ranging from 0 for a rough surface to 2 for a polished surface. In the paper, we set $k_{s}=1$, representing a

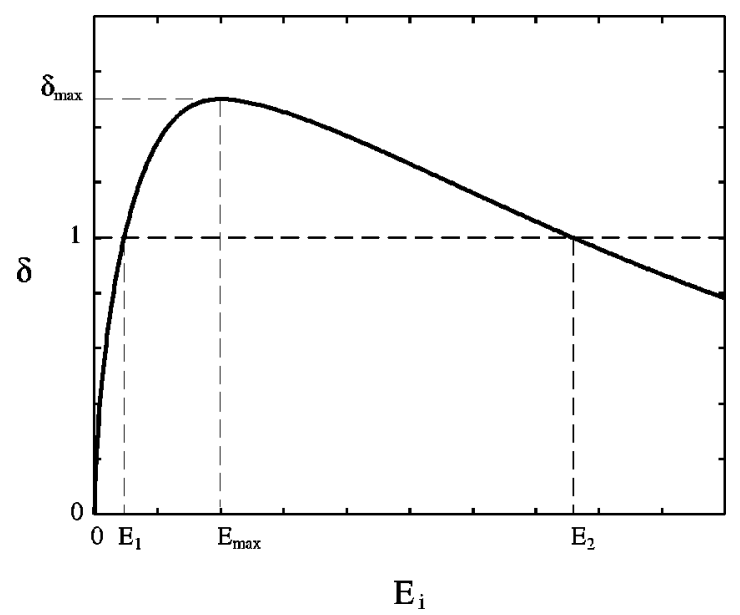

FIG. 2. Dependence of secondary electron yield, $\delta$, on impact energy, $E_{i}$. 
typical dull surface. For most dielectrics, $E_{\max 0}$ is of the order of a few hundred electron volts, see, e.g., Ref. 15.

By normalizing (time, velocity, electric field, and magnetic field) with respect to $\left(1 / \omega, \sqrt{E_{\max 0} / m}, \omega / e \sqrt{m E_{\max 0}}, m \omega / e\right)$, we write the normalized force equation of the secondary electrons as

$$
\frac{d \mathbf{v}}{d t}=-[\mathbf{E}+\mathbf{v} \times \mathbf{B}]
$$

Here, $\mathbf{E}=\left(E_{\mathrm{dc}}+E_{\mathrm{rf} 0} \sin (t+\theta) \sin \psi, E_{\mathrm{rf} 0} \sin (t+\theta) \cos \psi, 0\right), \mathbf{B}$ is the external magnetic field, $m$ is the electron mass, and $e$ is electron charge.

The distribution functions for the emission energy, and emission angle, for the secondary electrons are assumed to be of the form ${ }^{15,16}$

$$
\begin{aligned}
& f\left(E_{0}\right)=\frac{E_{0}}{E_{0 m}^{2}} e^{-E_{0} / E_{0 m},} \\
& g(\phi)=\frac{1}{2} \sin \phi,
\end{aligned}
$$

respectively. Note that $E_{0 m}$ is the peak of the emission energy distribution, and is estimated to be on the order of an electron volt. The mean emission energy is $2 E_{0 m}$.

In the simulation, a macroparticle is launched at $t=0$ from the surface $(x=0)$ according to the distributions in Eq. (4). It hits the surface after a time of flight $T$. Using the force equation and the parameters $\mathbf{B}, E_{\mathrm{rf} 0}, E_{\mathrm{dc}}, \theta, \phi$, and $\psi$, we solve for the trajectory $\left[\mathrm{v}_{x}(t), \mathrm{v}_{y}(t), \mathrm{v}_{z}(t)\right]$ and flight time $T$ (see the following). The impact energy, $E_{i}$, and the impact angle, $\xi$, are then given by

$$
\begin{aligned}
& E_{i}=\frac{1}{2}\left[\mathrm{v}_{x}^{2}(T)+\mathrm{v}_{y}^{2}(T)+\mathrm{v}_{x}^{2}(T)\right], \\
& \xi=\arctan \left(\sqrt{\frac{\mathrm{v}_{y}^{2}(T)+\mathrm{v}_{x}^{2}(T)}{\mathrm{v}_{x}^{2}(T)}}\right) .
\end{aligned}
$$

From $E_{i}$ and $\xi$, we calculate the secondary electron yield from Eqs. (1) and (2). We can determine either an exponentially growing or an exponentially decaying trend in the number of electrons in the avalanche, depending on the external parameters, such as $\mathbf{B}, E_{\mathrm{dc}}, E_{\mathrm{rf} 0}, \psi$, and $\delta_{\max 0}$. For any given values of the fields, the growth rate is determined by the average value of the secondary electron yield, averaged over the distributions of random emission energy, random emission angle, and random rf phase at emission (a uniformly random distribution is used for the $\mathrm{rf}$ phase, see also the end of Sec. III).

In this paper, we pick $\delta_{\max 0}=3$, and $E_{\max 0}=420 \mathrm{eV}$; then systematically vary both $E_{\mathrm{dc}}$ and $E_{\mathrm{rf} 0}$, and determine the boundaries of the multipactor region (exponential growth rate equals to zero) for different values of $\mathbf{B}$ and $\psi$.

Recalling the normalization parameter $m \omega / e$ used for the magnetic field, one realizes that the magnitude of the normalized magnetic field is equal to the ratio of the cyclotron frequency to the $\mathrm{rf}$ frequency $|\mathbf{B}|=\Omega=\omega_{c} / \omega$.
Here, we show the trajectories and the time of flight of the macroparticle subjected to $\Omega, E_{\mathrm{dc}}$ and $E_{\mathrm{rf} 0}$, of the three different magnetic field configurations.

(a) Magnetic field is parallel to $E_{\mathrm{rf}}\left(\vec{B}=\Omega_{y} \hat{y}\right)$ :

$$
\begin{aligned}
& \mathrm{v}_{x}(t)=\mathrm{v}_{0} \sin \phi \cos \left(\Omega_{y} t\right)-\frac{E_{\mathrm{dc}}}{\Omega_{y}} \sin \left(\Omega_{y} t\right), \\
& \mathrm{v}_{y}(t)=\mathrm{v}_{0} \cos \phi+E_{\mathrm{rf} 0}[\cos (\theta+t)-\cos \theta], \\
& \mathrm{v}_{z}(t)=\frac{E_{\mathrm{dc}}}{\Omega_{y}}\left[1-\cos \left(\Omega_{y} t\right)\right]-\mathrm{v}_{0} \sin \phi \sin \left(\Omega_{y} t\right), \\
& T=\frac{2}{\Omega_{y}} \arctan \left[\frac{\Omega_{y} \mathrm{v}_{0} \sin \phi}{E_{\mathrm{dc}}}\right] .
\end{aligned}
$$

(b) Magnetic field is parallel to $E_{\mathrm{dc}}\left(\vec{B}=\Omega_{x} \hat{x}\right)$ :

$$
\begin{aligned}
& \mathrm{v}_{x}(t)=\mathrm{v}_{0} \sin \phi-E_{\mathrm{dc}} t, \\
& \mathrm{v}_{y}(t)=\mathrm{v}_{0} \cos \phi \cos \left(\Omega_{x} t\right)+\frac{E_{\mathrm{rf} 0}}{\Omega_{x}^{2}-1}\left[\cos \theta \cos \left(\Omega_{x} t\right)\right. \\
& \left.-\cos (\theta+t)-\Omega_{x} \sin \theta \sin \Omega_{x} t\right], \\
& \mathrm{v}_{z}(t)=\mathrm{v}_{0} \cos \phi \sin \left(\Omega_{x} t\right)+\frac{E_{\mathrm{rf} 0}}{\Omega_{x}^{2}-1}\left[\cos \theta \sin \left(\Omega_{x} t\right)\right. \\
& \left.+\Omega_{x}\left(\sin \theta \cos \left(\Omega_{x} t\right)-\sin (\theta+t)\right)\right], \\
& T=\frac{2 \mathrm{v}_{0} \sin \phi}{E_{\mathrm{dc}}} \text {. }
\end{aligned}
$$

(c) Magnetic field is perpendicular to both $E_{\mathrm{dc}}$ and $E_{\mathrm{rf}}\left(\vec{B}=\Omega_{z} \hat{z}\right)$

$$
\begin{aligned}
\mathrm{v}_{x}(t)= & \mathrm{v}_{0} \sin \left(\phi-\Omega_{z} t\right)-\frac{E_{\mathrm{dc}}}{\Omega_{z}} \sin \left(\Omega_{z} t\right) \\
& +\frac{E_{\mathrm{rf} 0}}{\Omega_{z}^{2}-1}\left[\sin \left(\theta-\Omega_{z} t\right)+\Omega_{z} \sin (\theta+t)\right. \\
& \left.-\left(1+\Omega_{z}\right) \sin \theta \cos \left(\Omega_{z} t\right)\right],
\end{aligned}
$$

$\mathrm{v}_{y}=\mathrm{v}_{0} \cos \left(\phi-\Omega_{z} t\right)-\frac{E_{\mathrm{dc}}}{\Omega_{z}}\left[1-\cos \left(\Omega_{z} t\right)\right]$

$$
\begin{aligned}
& +\frac{E_{\mathrm{rf} 0}}{\Omega_{z}^{2}-1}\left[\cos \left(\theta-\Omega_{z} t\right)-\cos (\theta+t)\right. \\
& \left.-\left(1+\Omega_{z}\right) \sin \theta \sin \left(\Omega_{z} t\right)\right]
\end{aligned}
$$

$\mathbf{v}_{z}(t)=0$

$$
\begin{gathered}
\frac{E_{\mathrm{rf} 0}}{\Omega_{z}^{2}-1}\left[\frac{\cos \left(\theta-\Omega_{z} T\right)-\cos \theta-\left(1+\Omega_{z}\right) \sin \theta \sin \left(\Omega_{z} T\right)}{\Omega_{z}}\right. \\
\left.+\Omega_{z}[\cos \theta-\cos (\theta+T)]\right]+\frac{\mathrm{v}_{0}}{\Omega_{z}}\left[\cos \left(\phi-\Omega_{z} T\right)\right. \\
-\cos \phi]-\frac{E_{\mathrm{dc}}}{\Omega_{z}^{2}}\left[1-\cos \left(\Omega_{z} T\right)\right]=0 .
\end{gathered}
$$

In general, the time of flight of the macroparticle is inversely proportional to the dc charging field [see Eqs. (6) and 


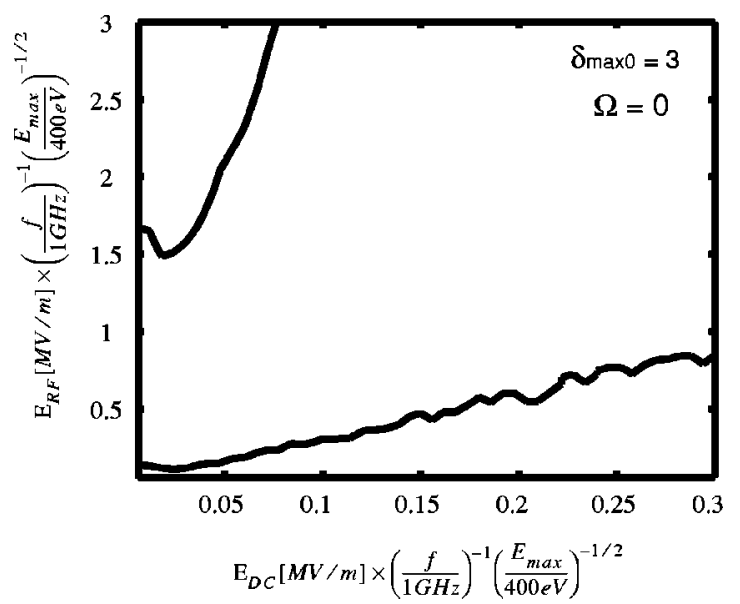

FIG. 3. Multipactor region boundaries in the plane of $\left(E_{\mathrm{dc}}, E_{\mathrm{r} f 0}\right)$ for $\delta_{\max 0}$ $=3$, with no magnetic field $(\Omega=0)$.

(7)]. However, it is more complicated for a perpendicular magnetic field case, which is solved numerically as the first positive root (other than zero) from Eq. (8). In the limit $\Omega$ $\rightarrow 0$, the equations become

$$
\begin{aligned}
& \mathrm{v}_{x}(t)=\mathrm{v}_{0} \sin \phi-E_{\mathrm{dc}} t, \\
& \mathrm{v}_{y}(t)=\mathrm{v}_{0} \cos \phi+E_{\mathrm{rf0}}[\cos (\theta+t)-\cos \theta], \\
& \mathrm{v}_{z}(t)=0, \\
& T=\frac{2 \mathrm{v}_{0} \sin \phi}{E_{\mathrm{dc}}},
\end{aligned}
$$

Equation (9) is exactly same as in the case of zero magnetic field. ${ }^{15}$

The trajectory and time of flight for the oblique rf case $(\Omega=0, \psi \neq 0)$ are given by

$\mathrm{v}_{x}=\mathrm{v}_{0} \sin \phi-E_{\mathrm{dc}} t+E_{\mathrm{rf} 0} \sin \psi(\cos (t+\theta)-\cos \theta)$,

$\mathrm{v}_{y}=\mathrm{v}_{0} \cos \phi+E_{\mathrm{rf} 0} \cos \psi(\cos (t+\theta)-\cos \theta)$,

$\left(\mathrm{v}_{0} \sin \phi-E_{\mathrm{rf} 0} \sin \psi \cos \theta\right) T-\frac{E_{\mathrm{dc}}}{2} T^{2}+E_{\mathrm{rf} 0} \sin \psi(\sin (T$

$+\theta)-\sin \theta)=0$.

If the time of flight exceeds $10 \mathrm{rf}$ periods for any particle, that particle is assumed to be lost.

\section{SUSCEPTIBILITY DIAGRAM}

Before discussing the effects of magnetic field, and oblique angle on the multipactor discharge on dielectric, let us review some of the key features in the zero magnetic field, nonoblique case. ${ }^{15}$ Figure $3^{20}$ shows the boundary region for multipactor discharge for this scenario. The lower and upper boundaries correspond to the two crossover points $\left(E_{1}\right.$ and $E_{2}$ in Fig. 2). The secondary electron yield is greater than unity only when the impact energy lies between $E_{1}$ and $E_{2}$. Changing the magnitude of the rf field changes the amount of energy gained for a given flight time. On the other hand, changing the dc field changes the time of flight, and hence also the amount of energy gained. If the rf field is too low or too high, the impact energy will be lower than $E_{1}$ or higher than $E_{2}$, at a fixed dc restoring field (or time of flight). This explains the existence of lower and upper boundaries. If the time of flight decreases by increasing the dc field, a larger $\mathrm{rf}$ field is needed to maintain $\delta=1$. This explains the existence of positive slope of the two boundaries. It is worth mentioning that this positive slope condition is always valid even in the cases with magnetic field, while in the case of oblique rf fields it is valid for high restoring fields.

For low values of the restoring field the time of flight may be equal to or greater than half a rf period. Any lengthening of the flight time beyond this value will not lead to greater impact energy, on average, as the electrons will simply decelerate or reverse at some point during there flight. This is seen in the leveling off of the susceptibility boundaries at low values of $\mathbf{E}_{\mathrm{dc}}$ in Fig. 3.

At high values of the dc field, the flight time is sufficiently short that the electrons see an essentially static accelerating field. Under these conditions the boundaries in the susceptibility diagram are linear asymptotically.

There are a few points to keep in mind when looking at the susceptibility diagram. First of all, the growth rate is averaged over all rf phases. This is a good method for determining susceptibility to multipactor, when the time of flight is on the order of half a rf period or longer, as it mirrors the random times of emission of the secondary electrons. For large values of the restoring field, the flight time of the electrons may be much less than half a rf period, and the uniform distribution in $\mathrm{rf}$ phase will not capture the essence of the actual distribution of secondary electrons. In this case the growth rate oscillates in time with a frequency equal to the rf frequency. The boundaries of the susceptibility diagrams presented in this paper are calculated using the average growth rate over a rf period. If stray electrons are incident upon the dielectric at such a rf phase that the instantaneous growth rate is positive, although it is on average zero, one may well see multipactor discharge initiate, last for some fraction of an rf period, and then diminish. This drawback of the random phase assumption for high values of the charging field is not that critical, since prior to any multipactor discharge taking place the dielectric will not be highly charged. One is mainly interested in the high dc field part of the lower boundary when considering saturation levels, ${ }^{14}$ in which case it may be used to yield a useful average.

\section{MAGNETIC FIELD}

By systematically increasing the magnetic field's magnitude $\Omega$, from zero up to one (at about rf frequency), we examine the change of the lower and upper boundaries. Figure 4 shows the susceptibility diagram of (a) $\mathbf{B}=\Omega_{y} \hat{y}$, (b) $\mathbf{B}=\Omega_{x} \hat{x}$, and (c) $\mathbf{B}=\Omega_{z} \hat{z}$, for various value of $\Omega$. Here the subscripts $x, y, z$ denote the direction of the magnetic field. In all cases, only the magnitude of $\Omega$ is important. The sign of $\Omega$ does not make any difference in the susceptibility diagram.

With a magnetic field parallel to the $\operatorname{rf}$ field, $\mathbf{B}=\hat{\mathbf{y}} \Omega_{y}$, we have gyromotion in the $x-z$ plane. However, this gyromotion can not gain any energy from the rf field, as the rf 

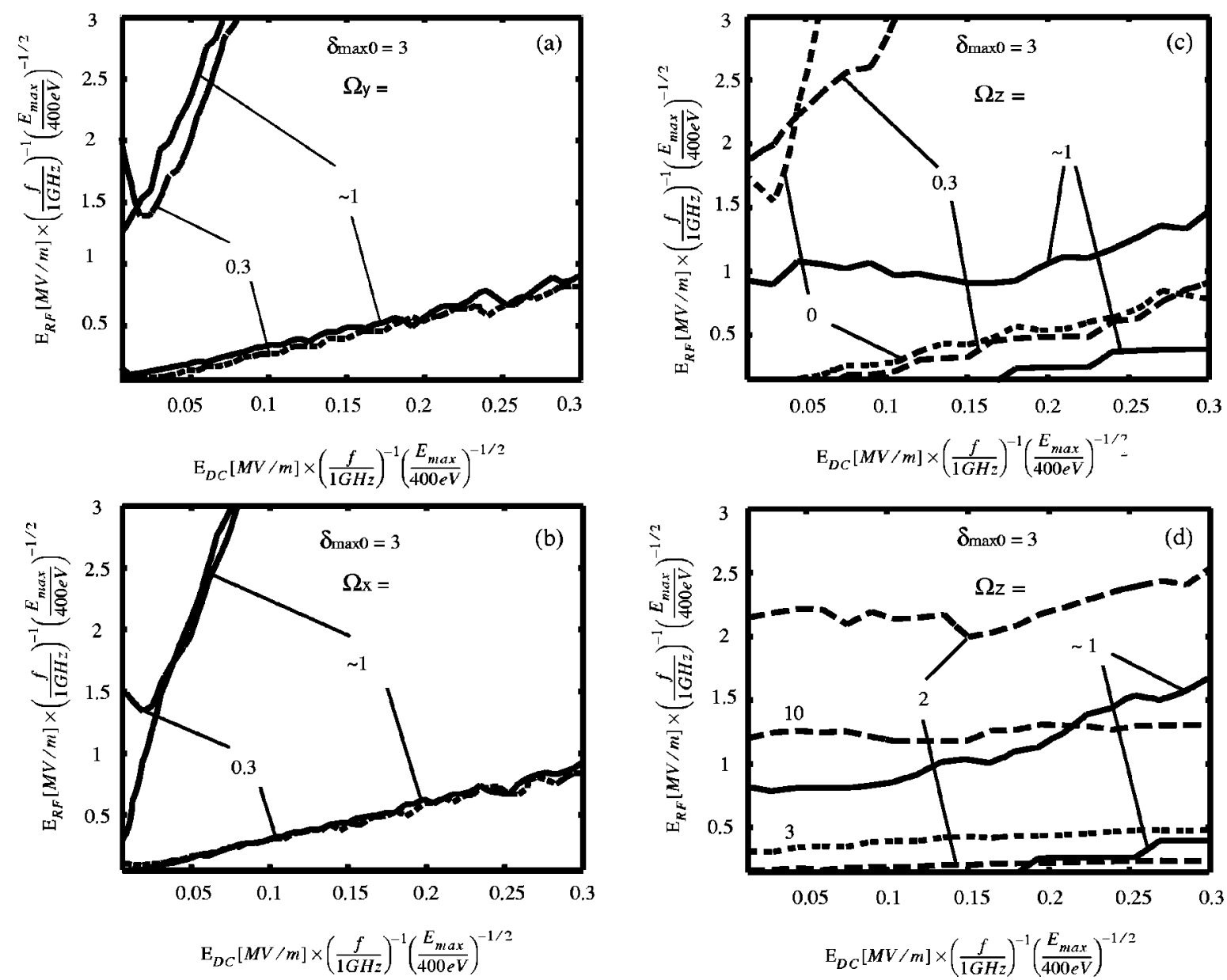

FIG. 4. Multipactor region boundaries in the plane of $\left(E_{\mathrm{dc}}, E_{\mathrm{rf} 0}\right)$ for $\delta_{\max 0}=3$. (a) with an external magnetic field parallel to rf electric field for $\Omega_{y}=0.3$ (dash line) and 1 (solid line). (b) With an external magnetic field parallel to dc electric field, for $\Omega_{x}=0.3$ (dash-line) and 0.99 (solid line). (c) With an external magnetic field perpendicular to both rf and dc electric field, for $\Omega_{z}=10^{-6}$ (dot line), 0.3 (dash line), and 0.99 (solid line). (d) With an external magnetic field perpendicular to both rf and dc electric field, for $\Omega_{z}=0.99$ (solid line), 2 (dash line), 3 (dot line), and 10 (dash-dot line). Note that the upper boundaries for $\Omega_{z}=3$ and 10 are beyond the range of the graph.

field is perpendicular to the motion. In the limit of small $\Omega_{y}$, the time of flight recovers to $T_{0}$ [see Eq. (6)]. Here, $T_{0}$ $=2 \mathrm{v}_{0} \sin \phi / E_{\mathrm{dc}}$ is defined as the time of flight with no magnetic field throughout this paper. Varying $\Omega_{y}$ from 0 to about 1 , we found that the effect of $\Omega_{y}$ is negligible. Figure 4(a) gives the typical boundaries at $\Omega_{y}=0.3$ and 1.0. The boundary region of multipactor discharge is qualitatively similar to the case of no magnetic field (see Fig. 3). Thus a magnetic field that is parallel to the rf field does not change the susceptibility diagram.

A magnetic field that is parallel to the dc field, $\mathbf{B}$ $=\hat{\mathbf{x}} \Omega_{x}$ gives a gyromotion in the $y-z$ plane. Since the gyromotion is in the $y-z$ plane, the motion in the $x$ direction governs the time of flight, and it is equal to $T_{0}$ [see Eqs. (7) and (9)]. With the same amount of flight time, secondary electrons gain more energy (compared to the case without magnetic field) from the $\mathrm{rf}$ field through the gyromotion in $y-z$ plane, and from $E_{\mathrm{rf}} \times \Omega_{x}$ drift. We could anticipate that resonant absorption occurs at $\Omega_{x}=1$, when the cyclotron frequency is equal to the rf frequency. For resonant absorption to become apparent, the time of flight must be sufficiently long (on the order of a rf period or longer). Therefore, the susceptibility diagram is altered only at the extremely low end of the dc field magnitude, where resonance manifests itself as a lowering of the susceptibility boundaries. Figure 4(b) gives the typical boundaries at $\Omega_{x}=0.3$ and 1.0 diagram.

In the last case, we have a magnetic field perpendicular to both the $\mathrm{rf}$ and dc fields $\mathbf{B}=\hat{\mathbf{z}} \Omega_{z}$ [Fig. 4(c)]. The trajectory and time of flight are complicated functions of $E_{\mathrm{rf0}}, E_{\mathrm{dc}}$, $\Omega_{z}, \mathrm{v}_{0}, \theta$, and $\phi$. From force considerations, it is apparent that the magnetic field does not play an important part in restoring electrons to the surface unless the magnetic force component, $\Omega_{z} \mathrm{v}_{y}$, is comparable in magnitude to the force exerted by the dc electric field $E_{\mathrm{dc}}$. Therefore the effect of the external magnetic field is first realized for the upper boundary of the susceptibility diagram, where the typical velocity of the electrons along their trajectory is much higher, because of the higher impact energy (a hundred times higher), than the velocities typical of the lower boundary. Up to a value of $\Omega_{z}=1$, the upper boundary is lowered, reaching a minimum value around $\Omega_{z}=1$. This minimum occurs when a portion of the electrons emitted from the surface are not pulled immediately back to the surface, but remain in flight for up to a few rf periods. This leads to a resonant condition where these electrons pick up much more energy than they 
would in the absence of the magnetic field, for given values of $E_{\mathrm{rf0}}$ and $E_{\mathrm{dc}}$. For these resonating electrons, a much lower value of the tangential field, $E_{\mathrm{rf} 0}$, is needed to reach the first crossover energy. Of course, for a portion of the secondaries emitted, the magnetic field will reduce their time of flight, leading to less energy gain, but this reduction is of a smaller scale than the energy increase for the resonant electrons. Hence, on average, the upper susceptibility boundary is lowered around the resonant condition, $\Omega_{z}=1$. As the magnetic field strength is increased, these resonance effects vanish, and the magnetic force only leads to a drastic reduction of the time of flight (or equivalently to a shortening of the flight path). An increasing magnetic field now has the effect to reduce the amount of energy given to a particle in a bounce, for given values of $E_{\mathrm{rf0}}$ and $E_{\mathrm{dc}}$ [Fig. 4(d)]. Hence increasing the magnetic field strength raises the boundaries of the susceptibility diagram. This raising even becomes apparent for the lower boundary, as for strong magnetic fields the magnetic force component, $\Omega_{z} \mathrm{v}_{y}$, becomes comparable to the dc restoring field, $E_{\mathrm{dc}}$. In Fig. 4(d), the upper boundary of the susceptibility diagram for the high magnetic field cases $\Omega_{z}$ $=3$ and 10 are beyond the scales of the diagram.

From the susceptibility diagrams shown in Fig. 4, the presence of an external magnetic field does not qualitatively change the susceptibility diagram if it is parallel to $\mathbf{E}_{\mathrm{rf}}$. The external magnetic field lowers the susceptibility boundaries for low values of the dc electric field, when $\mathbf{B}$ is parallel to $\mathbf{E}_{\mathrm{dc}}$. This statement holds for all magnetic fields simulated, up to those values whose cyclotron frequency is on the order of the rf frequency. However, magnetic field perpendicular to both the rf field and the dc field can change the susceptibility diagram considerably for cyclotron frequencies close to or greater than the rf frequency. The magnetic field strength, $B_{\text {res }}$ which gives a cyclotron frequency equal to the rf frequency, is given by

$$
B_{\text {res }}[T]=0.036 f(\mathrm{GHz}),
$$

where $f$ is the rf frequency. There is no appreciable effect if $B_{z}$ is less than $B_{\text {res }}$. We now digress to comment on the role of the rf magnetic field, ${ }^{18}$ assuming the absence of an external $\mathbf{B}$ field, and assuming a normally incident plane wave with no reflection. Our calculations show a negligible change to the lower boundary, while the upper boundary is greatly extended (to such an extent that it may be considered to be eliminated in some cases). This is readily explained. The normalized rf magnetic field is $\Omega=\Omega_{\mathrm{rf}} \hat{\mathbf{z}}$, with

$$
\Omega_{\mathrm{rf}}=-\frac{1}{c} E_{\mathrm{rf} 0} \sin (t+\theta),
$$

where $c$ is the normalized speed of light in vacuum ( $c$ $\left.=\sqrt{511 \mathrm{keV} / E_{\max 0}(\mathrm{eV})}\right)$. For the lower bound of the susceptibility diagram (corresponding to the first crossover energy of the secondary emission curve) one typically has $\mathrm{V} / \mathrm{c}$ $\approx 1 \%$, and $E_{\mathrm{rf} 0} / E_{\mathrm{dc}} \approx 2-3$. Hence the magnetic force is negligible compared to the dc electric field force at the lower boundary. Therefore the lower boundary remains unchanged. For impact energies corresponding to the second crossover energy one typically has $\mathrm{V} / c \approx 10 \%$ if $\delta_{\max 0} \geqslant 3$. The value of $E_{\mathrm{rf0}} / E_{\mathrm{dc}}$ will also be quite high. In this region the rf mag- netic force is significant, and may even be dominant in comparison to the electrostatic force due to the charging field. There is no resonant effect as in the case of the external magnetic field, and the only effect of the rf magnetic field is to shorten the flight trajectory. As the flight time is typically very short, one may understand the situation by approximating the electric field tangential to the dielectric to be constant. For large values of $E_{\mathrm{rf} 0} / E_{\mathrm{dc}}$ one may further ignore the effects of the dc field. In this case the distance the electrons travel along the dielectric is of the order of the Larmor radius, and scales as $1 / E_{\mathrm{rf}}$. The energy added to the electrons in flight is the product of the tangential field and the distance traveled tangentially. The energy gain is roughly approximated by

$$
\Delta \text { Energy }=\int_{\text {Bounce }} \mathbf{E} \cdot d \mathbf{s} \sim E_{\mathrm{rf}} \frac{1}{E_{\mathrm{rf}}}=\text { constant. }
$$

This leads to saturation in the energy gained per bounce. If that energy level is lower than the second crossover energy, the upper susceptibility boundary is eliminated.

It should be noted that the rf magnetic field effects may be neglected in the presence of an external magnetic field, $\Omega_{z}$, of magnitude $\Omega_{z} \gg E_{\mathrm{rf} 0} / c$, where $c$ is the normalized speed of light (typically around 30). Note also that the effects of the rf magnetic field upon the upper boundary are unimportant for dielectrics with low secondary yields $\left(\delta_{\max 0}\right.$ $\leqslant 1.5$ ), since, in these cases, the second crossover energy is substantially reduced, leading to a low $\mathbf{v} \times \mathbf{B}_{\mathrm{rf}}$ force.

\section{OBLIQUE rf FIELD}

An oblique rf electric field has a component perpendicular to the dielectric, which can either reinforce or reduce the restoring field, depending on the rf phase. Thus the time of flight of the electrons in the discharge can be strongly affected. From previous analysis ${ }^{15}$ it has been found that the flight time of the electrons is of greatest importance in determining the susceptibility diagram for multipactor discharge. Figure 5 shows susceptibility diagrams for various angles of obliqueness.

Simulations reveal that the first crossover energy still determines the lower boundary. It is not greatly altered, even though its slope decreases slightly with increasing angle of obliqueness. The contribution to the average secondary emission coefficient from electrons emitted when the restoring field is weakened, due to the rf contribution, is the reason for this decrease of the slope, as it outweighs the effects of the electrons emitted when the rf field adds to the restoring field. To understand this better recall that for sufficiently large values of the restoring field, $\mathbf{E}_{\mathrm{dc}}+\mathbf{E}_{\mathrm{rf0}} \sin \psi \cos (\omega t+\phi)$, the rf field changes very little during the electrons' time of flight. Therefore one may use the approximation that the rf field is constant throughout the time of flight. Hence one may approximately find the boundary of the susceptibility curve by calculating the secondary emission obtained with a perpendicular field of $\left|\mathbf{E}_{\mathrm{dc}}+\mathbf{E}_{\mathrm{rf0}} \sin \psi \cos (\phi)\right|$ and a parallel field of $\left|\mathbf{E}_{\mathrm{rf} 0} \cos \psi \cos (\phi)\right|$, and averaging over the phase, $\phi$, with a uniform distribution from 0 to $2 \pi$. Figure 6 shows a typical 

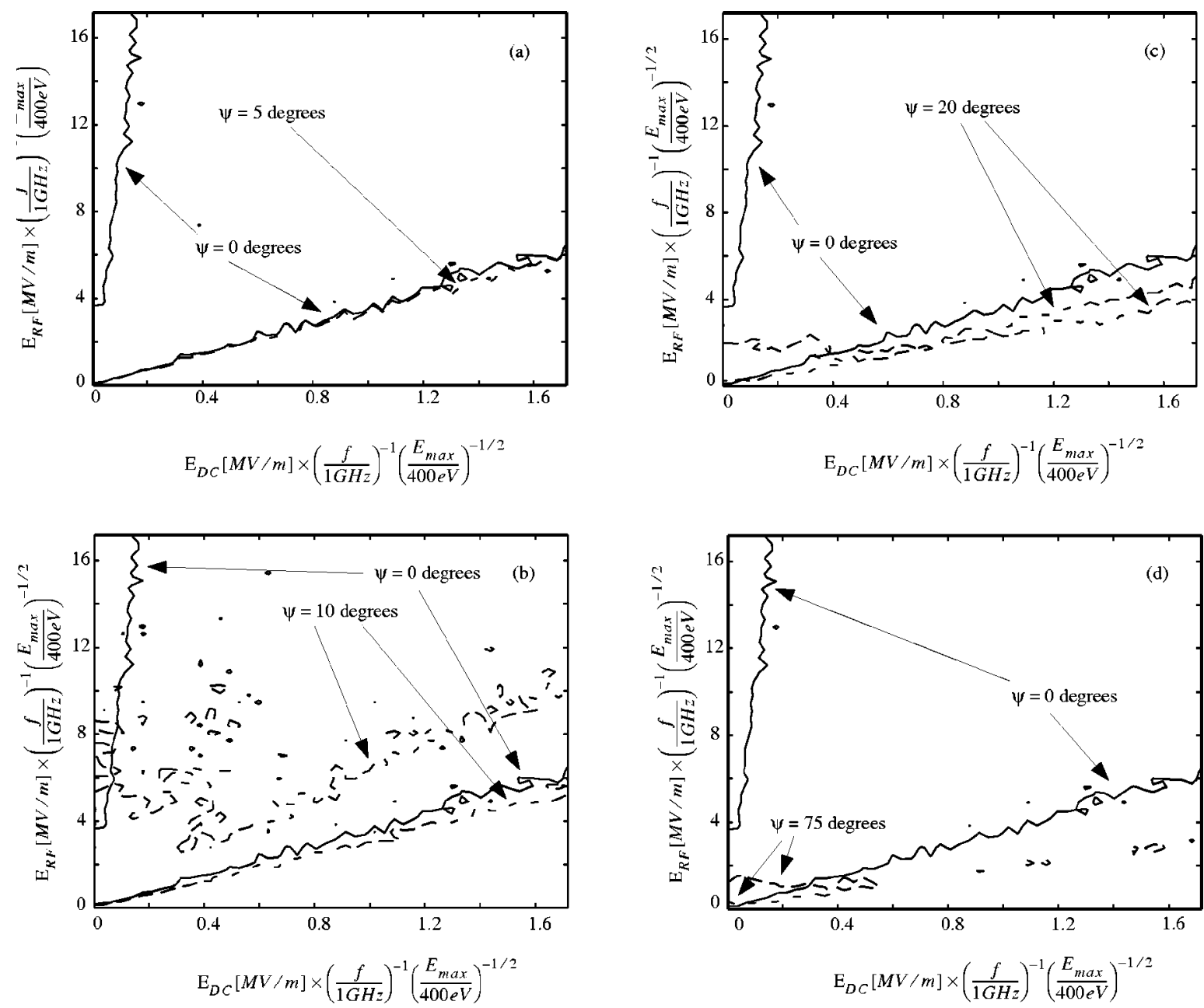

FIG. 5. Susceptibility boundaries in the $\left(E_{\mathrm{dc}}, E_{\mathrm{rf0}}\right)$ for $\delta_{\max 0}=3$, and various angles of obliqueness. (a) $\psi=0^{\circ}$ (solid line), $5^{\circ}$ (dash line), note that the upper boundary for $\psi=5^{\circ}$ is beyond the range of the graph; (b) $\psi=0^{\circ}$ (solid line), $10^{\circ}$ (dash line); (c) $\psi=0^{\circ}$ (solid line), $20^{\circ}$ (dash line); (d) $\psi=0^{\circ}$ (solid line), $75^{\circ}$ (dash line).

graph of the secondary yield as a function of constant tangential electric field, $E_{t}$, and constant restoring field, $E_{r}$, perpendicular to the surface, throughout the time of flight (i.e., dc conditions). The lower boundary of the susceptibility diagram corresponds to the higher restoring field at which $\delta=1$ in Fig. 6. Because of the decreasing slope of the secondary emission curve shown in Fig. 6 it is clear that, given a fixed tangential field strength, the average secondary emission for restoring fields of magnitude $\mid \mathbf{E}_{\mathrm{dc}}$ $+\mathbf{E}_{\mathrm{rf} 0} \sin \psi \cos (\phi) \mid$ and $\left|\mathbf{E}_{\mathrm{dc}}-\mathbf{E}_{\mathrm{rf} 0} \sin \psi \cos (\phi)\right|$ will be greater than the secondary emission for a restoring field of $\left|\mathbf{E}_{\mathrm{dc}}\right|$. This explains the lowering of the slope of the lower susceptibility boundary in the presence of an oblique field.

There are two main changes to the upper susceptibility boundary in the presence of an oblique field, from that of the nonoblique case. First of all, for small angles of obliqueness $\left(\psi \lesssim 5^{\circ}\right)$, the low- $E_{\mathrm{dc}}$ end of the susceptibility region is extended to higher values of $E_{\mathrm{rf}}$ [beyond the abscissa of the graph in Fig. 5(a)]. This is due to the fact that at certain phases of the rf field, the restoring field is greatly increased, shortening the flight time enough to bring the impact energy to within the second crossover energy. For larger angles, $\psi$, this extension vanishes as the contribution of electrons emit- ted at large restoring fields diminishes, as compared with the losses when the rf field is pushing the electrons away from the surface of the dielectric. At higher values of $E_{\mathrm{dc}}$ and for angles of obliqueness $\Omega \gtrsim 5^{\circ}$, one sees a drastic decline of the slope of the upper boundaries, leading to a narrowing of the susceptibility region [Figs. 5(b)-5(d)]. In this case the electrons emitted during a rf phase, such that the restoring field is greatly reduced, have a greater effect on the average growth rate, than do those emitted during a rf phase that leads to a shorter flight time.

The results described in this section are obtained in the absence of electromagnetic effects. An obvious question arises; what happens if the rf magnetic field is included. One may estimate this by looking at the relative magnitudes of the components of the forces acting perpendicular to the dielectric surface. At the lower boundary the magnetic field force is negligible (see Sec. IV). At the upper boundary the magnetic force is given by, $\left(\mathrm{v}_{y} / c\right) E_{\mathrm{rf} 0} \sin (t+\theta)$, While the force due to the oblique field is given by $\sin \psi E_{\mathrm{rf} 0} \sin (t+\theta)$. At the upper boundary, typically $\mathrm{v} / c \approx 0.1$, so that for angles of obliqueness greater than $5^{\circ}-10^{\circ}$ the oblique field effects become dominant. 


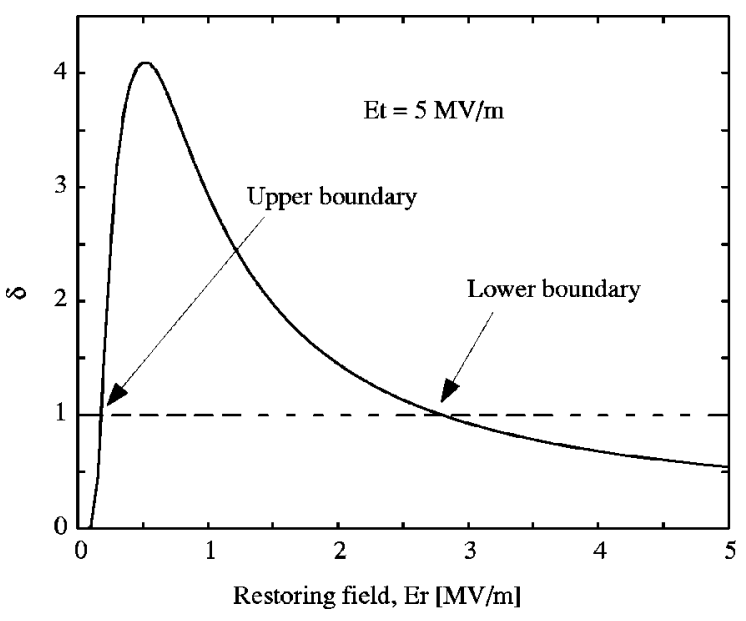

FIG. 6. Secondary electron yield as a function of restoring field under dc conditions. $\quad \delta_{\max 0}=3, \quad E_{\max 0}=420 \mathrm{eV}$, tangential field strength $E_{t}$ $=5 \mathrm{MV} / \mathrm{m}$, normal emission with emission energy of $2 \mathrm{eV}$.

\section{CONCLUDING REMARKS}

In this paper, we find that external magnetic fields do not appreciably affect susceptibility to multipactor discharge, unless the applied magnetic field is parallel to the dielectric surface, and perpendicular to the rf electric field. The upper boundary of the susceptibility diagram is lowered, via resonance effects, at a magnetic field strength of $B_{\text {res }}[T]$ $\approx 0.036 f(\mathrm{GHz})$, where $f$ is the $\mathrm{rf}$ frequency. The upper boundary of the susceptibility diagram may be raised for magnetic field strengths of $B_{z} \gg B_{\text {res }}$. The rf magnetic field does not affect the lower boundary of the susceptibility diagram, but may raise the upper boundary greatly. Since saturation occurs on the lower boundary, ${ }^{16}$ the rf magnetic field has only minimal effects on the saturation mechanism.

We also find that a dielectric is most susceptible to single surface multipactor when the obliqueness of the $\mathrm{rf}$ field is less than approximately $5^{\circ}$. For larger angles the area of susceptibility is greatly reduced.

\section{ACKNOWLEDGMENTS}

We wish to thank Professor Andreas Neuber ${ }^{18}$ for reminding us of the potential importance of the rf magnetic field on high-energy multipactoring electrons. This work was supported by the Department of Energy, by the Multidisciplinary University Research Initiative (MURI), managed by the Air Force Office of Scientific Research and subcontracted through Texas Tech University, and by the Northrop Grumman Industrial Associated Program.

${ }^{1}$ J. R. M. Vaughan, IEEE Trans. Electron Devices 15, 883 (1968); 35, 1172 (1988).

${ }^{2}$ D. H. Preist and R. C. Talcott, IRE Trans. Electron Devices 8, 243 (1961).

${ }^{3}$ J. R. M. Vaughan, IEEE Trans. Electron Devices 8, 302 (1961).

${ }^{4}$ S. Yamaguchi, Y. Saito, S. Anami, and S. Michizono, IEEE Trans. Nucl. Sci. 39, 278 (1992).

${ }^{5}$ R. A. Rimmer, Ph.D. dissertation, University of Lancaster, England, 1988.

${ }^{6}$ N. Rozario, H. F. Lenzing, K. F. Reardon et al., IEEE Trans. Microwave Theory Tech. 42, 558 (1994).

${ }^{7}$ R. A. Kishek, Ph.D. dissertation, University of Michigan, Ann Arbor, 1997.

${ }^{8}$ R. A. Kishek, Y. Y. Lau, L. K. Ang, A. Valfells, and R. M. Gilgenbach, Phys. Plasmas 5, 2120 (1998).

${ }^{9}$ R. A. Kishek and Y. Y. Lau, Phys. Rev. Lett. 75, 1218 (1995); Phys. Plasmas 3, 1481 (1996).

${ }^{10}$ S. Riyopoulos, D. Chernin, and D. Dialetis, Phys. Plasmas 2, 3194 (1995).

${ }^{11}$ S. Riyopoulos, D. Chernin, and D. Dialetis, IEEE Trans. Electron Devices 44, 489 (1997).

${ }^{12}$ S. Riyopoulos, Phys. Plasmas 4, 1448 (1997).

${ }^{13}$ R. A. Kishek, Y. Y. Lau, and D. Chernin, Phys. Plasmas 4, 863 (1997).

${ }^{14}$ A. Valfells, R. A. Kishek, and Y. Y. Lau, Phys. Plasmas 5, 300 (1998).

${ }^{15}$ R. A. Kishek and Y. Y. Lau, Phys. Rev. Lett. 80, 193 (1998).

${ }^{16}$ L. K. Ang, Y. Y. Lau, R. A. Kishek, and R. M. Gilgenbach, IEEE Trans. Plasma Sci. 26, 290 (1998).

${ }^{17}$ A. Neuber, J. Dickens, D. Hemmert, H. Krompholz, L. L. Hatfield, and M. Kristiansen, IEEE Trans. Plasma Sci. 26, 296 (1998).

${ }^{18}$ A. Neuber, D. Hemmert, H. Krompholz, L. L. Hatfield, and M. Kristiansen, J. Appl. Phys. 86, 1724 (1999).

${ }^{19}$ J. R. M. Vaughan, IEEE Trans. Electron Devices 36, 1963 (1989).

${ }^{20}$ The relationship between the values for the normalized electric field strengths, $E_{\text {norm }}$, and the values on the abscissae and ordinates of the susceptibility diagrams is given by $E(\mathrm{MV} / \mathrm{m}) / f(\mathrm{GHz}) \sqrt{\left(E_{\max 0}[\mathrm{eV}] / 400\right)}$ $=0.3 E_{\text {norm }}$, where $E_{\max 0}$ is the energy at which the secondary emission curve is at a maximum for normally incident electrons. 